\title{
Ofertas Terapéuticas, Estilos de Vida y "Cura Gay" en Chile: Aprendiendo a Ser Heterosexuales
}

\author{
Therapeutic Offers, Lifestyles, and "Gay Cure" in Chile: \\ Learning to Be Heterosexual
}

\author{
Tomás Ojeda \\ London School of Economics and Political Science
}

\begin{abstract}
El debate en torno a la llamada cura gay y las prácticas reparativas o de acompañamiento para individuos que desean cambiar su identidad sexual continúa presente. El artículo propone una aproximación crítica al lugar político de la psicoterapia en el debate actual sobre las ofertas de cambio de las sexualidades no heterosexuales y las formas de articulación de este debate en el contexto chileno. Para ello se discuten 2 dimensiones analíticas específicas. Primero, se revisan algunos de los principales hitos que posibilitaron la invención clínica del exgay y del dispositivo reparativo, discutiendo críticamente el lugar que ocupan la motivación, el esfuerzo y la libertad individual en el contexto en que se comprende actualmente la psicoterapia, particularmente, en referencia a lógicas de responsabilidad individual que serían propias del mercado y del neoliberalismo. En segundo lugar, se analiza el componente ideológico del dispositivo y el carácter contingente de la norma heterosexual, cuestionando la relativa legitimidad que tienen los discursos patologizantes de las sexualidades no heterosexuales en un contexto de disputa político-religiosa sobre los principios normativos que fundan el orden sexual contemporáneo.
\end{abstract}

Palabras clave: heteronormatividad, oferta terapéutica, patologización de la homosexualidad, terapias reparativas

The debate around the so-called gay cure and reparative practices or therapeutic accompaniment to individuals who want to change their sexual identity is still active. This article proposes a critical approach to the political place of psychotherapy in current debates around therapeutic offers aimed at changing non-heterosexual sexualities, focusing on the ways this discussion has played out in Chile. With this in mind, 2 specific analytical dimensions are discussed. Firstly, the article reviews some of the main milestones that enabled the clinical invention of the ex-gay and the reparative device, critically discussing the place that motivation, effort, and individual freedom play in the context in which psychotherapy is currently practiced, particularly in relation to logics of personal responsibility that are characteristic of the market and neoliberalism. Secondly, the ideological component of the device and the contingent nature of the heterosexual norm are also analysed, challenging the relative legitimacy of pathologising discourses against non-heterosexual sexualities, in a context where the normative principles of the contemporary sexual order are being contested by politico-religious forces.

Keywords: heteronormativity, therapeutic offer, pathologisation of homosexuality, reparative therapies

El debate en torno a la llamada cura gay y las prácticas reparativas, de acompañamiento o de conversión para individuos que quieren curarse de su atracción sexual no deseada al mismo sexo (AMS) continúa presente. Pese a que la homosexualidad dejó de ser considerada una patología en 1973 y que recientemente la Organización Mundial de la Salud (OMS) desclasificó la categoría "incongruencia de género" del capítulo de trastornos mentales de su manual diagnóstico CIE-11 (World Health Organization, 2018), aún existen profesionales de la salud mental que insisten en el estatuto patológico de la homosexualidad y las subjetividades trans, así como también en sus posibilidades de cura a través del acompañamiento, el coaching, la consejería o la psicoterapia (Colegio de Psicólogos de Chile, 2015a, 2015b; ver también Barrientos, Díaz, Gómez \& Muñoz, 2012).

Aun cuando el descrédito y el rechazo hacia este tipo de intervenciones son mayoritarios, la discusión pública tiende a centrarse casi exclusivamente en sus posibilidades de regulación, especialmente cuando se conoce algún caso o se sabe de alguna denuncia a través de los medios de comunicación. El foco del debate, a su vez, se desplaza desde un principio de no patologización hacia otro que problematiza distintos tipos de

Tomás Ojeda, Department of Gender Studies, London School of Economics and Political Science, Reino Unido.

El artículo recoge parte de las reflexiones contenidas en la tesis de Magíster en Psicología Clínica de Adultos, línea de especialización en Psicoanálisis de la Universidad de Chile, "El lugar de la cura en la terapia reparativa con personas homosexuales. Análisis crítico de las condiciones de emergencia del dispositivo reparativo y sus implicancias sobre el trabajo clínico con personas homosexuales".

La correspondencia relativa a este artículo debe ser dirigida a Tomás Ojeda, Department of Gender Studies, London School of Economics and Political Science, Houghton Street, London, WC2A 2AE, United Kingdom. E-mail: t.i.ojeda@lse.ac.uk 
evidencias en torno al cambio, que abarcan desde investigaciones científicas hasta testimonios de sobrevivientes y homosexuales sanados (Waidzunas, 2015). Esto último ha terminado por redefinir los términos de la discusión a una en la que se disputan distintos tipos de libertades — de expresión, de elegir, de enseñar-, multiplicándose los argumentos y las fuentes autorizadas para hablar sobre estos temas, destacando, entre otros, los colectivos de lesbianas, gais, bisexuales, trans e intersex (LGBTI), la sociedad civil, los medios de comunicación y prensa y los líderes religiosos y políticos.

Por otra parte, la creciente politización de las normas sexuales y su consiguiente efecto de desnaturalización del orden sexual contemporáneo (Fassin, 2006/2012) ha sido activamente resistida por organizaciones civiles y religiosas que reivindican el estatuto natural de la norma heterosexual. En el campo de la salud mental y la psicoterapia, estos grupos han buscado reforzar el carácter patológico de la homosexualidad y validar una terapéutica que busca curar y/o revertir su supuesta desviación (Cornejo, 2011; Wilkinson, 2013). Lo anterior ha facilitado la diseminación de nuevas argumentaciones y apoyos que funcionan con relativa efectividad en sistemas político-económicos neoliberales, como los que rigen en gran parte de los países latinoamericanos, especialmente en Chile.

Parafraseando al sociólogo francés Eric Fassin (2006/2012), el discurso reparativo y/o de tratamiento de la AMS aparecería en este contexto como una estrategia de tipo política que busca, entre otras cosas, devolverle a la norma heterosexual su poder inmanente en tanto principio organizador de lo social. Siguiendo con este argumento, el auge de las campañas antigénero a nivel global, junto con la lucha contra la denominada ideología de género por parte del activismo religioso conservador, son algunas de las formaciones políticas que han ido activándose en respuesta al avance de los movimientos LGBTI y feministas en Latinoamérica, quienes se han manifestado contra el carácter opresivo del patriarcado y la violencia homo, lesbo y transfóbica (Corrêa, 2018; Kuhar \& Paternotte, 2017; Morán Faúndez, 2012; Viveros Vigoya \& Rodríguez Rondón, 2017). Por tanto, un análisis sobre la vigencia de los discursos reparativos en los ámbitos políticos y de salud no solo debe limitarse a exponer sus falencias en los niveles de la evidencia y no efectividad. También debe preguntarse por las condiciones históricas y socioculturales que hacen posible que hoy sigamos discutiendo sobre estos temas, pese a los consensos institucionales en torno a la despatologización de las sexualidades no heterosexuales.

Con todo, este artículo busca intervenir en estos asuntos interrogando críticamente la vigencia de lo que Annie Wilkinson (2013) ha denominado prácticas reparativas en el debate actual sobre los vínculos entre homosexualidad, psicopatología y psicoterapia en el contexto latinoamericano, específicamente chileno. Para ello, presentaré algunos de los hitos que facilitaron la invención del exgay y de la terapia reparativa, utilizando la estructura de análisis descrita por Wilkinson (2013) en su investigación sobre las prácticas reparativas en Ecuador, en conjunto con investigaciones recientes sobre la medicalización de la homosexualidad en Chile. Si bien la literatura disponible sobre la patologización de la homosexualidad es de una riqueza difícil de captar en su complejidad en este texto (ver, por ejemplo, Bayer, 1987; Contardo, 2011; Cornejo, 2011; Figari, 2012; Wilkinson, 2013), presentaré una lectura particular de dicha historia a partir de intervenciones queer y feministas que han permitido repensar el fenómeno de la patologización en su impronta reguladora y normalizadora.

En un segundo momento, analizaré dos elementos de contexto que ayudan a comprender algunos de los motivos que explican la relativa vigencia del debate en torno a la cura gay en Chile: (a) la creciente mercantilización de la psicoterapia y las influencias del neoliberalismo en las formas en que se ofertan estilos de vida saludables bajo el paradigma de la libertad de elección, el esfuerzo individual y la motivación y (b) la reivindicación de un determinado orden sociosexual que restituye el poder de la norma heterosexual y sus efectos sobre la patologización de las diferencias.

\section{La Invención de lo Reparativo y la Figura del Exgay}

\section{El Poder Patologizante}

Desde principios del siglo XX, la psiquiatría y la psicología han participado activamente tanto en los procesos de normalización de la sexualidad como en su regulación institucional a través de distintos dispositivos, entre ellos, la creación del Comité de Nomenclatura y Estadística de la Asociación Estadounidense de Psiquiatría (APA) el año 1917 (Corrêa, Davis \& Parker, 2014). Dicha institucionalidad ilustra de buena forma cómo opera el poder a través del imperativo de la medicalización, el cual, según Foucault (1976/2011), no solo define a la sexualidad como un dominio susceptible de ser atravesada por 
procesos patológicos, sino también como "una vía de paso para las relaciones de poder" (p. 97) que demanda intervenciones de tipo terapéuticas y de normalización.

La hipótesis foucaultiana sobre la medicalización de la sexualidad y su correspondiente patologización ha sido retomada y enriquecida desde los estudios queer y feministas. Desde esta perspectiva, la patologización opera como una forma de control social que vuelve inteligibles las experiencias humanas no médicas dentro de los pares binarios normal/salud y anormal/enfermedad (Borba, 2017; Cornejo, 2011; Russo, 2004/2013). En términos generales, este mecanismo autoriza que profesionales de la salud mental y del área médica decidan qué cuerpos y existencias se considerarán legítimos, transformando, de paso, expresiones potencialmente neutras de diferencia (racial, sexual y de género) en jerarquías sociales opresivas y desiguales (Latham, 2017). En este contexto, la patologización de las sexualidades no normativas —en este caso, no heterosexuales - operaría excluyendo a algunas subjetividades del campo de lo inteligible (Suess, 2014), el cual, siguiendo a la filósofa feminista Judith Butler (1990/2007), estaría determinado por una matriz cultural de sentido que organiza la identidad personal "a través de los conceptos estabilizadores de sexo, género y sexualidad" (p. 71).

A partir de esta lógica, el poder patologizante lo entiendo aquí como un mecanismo de regulación que mantiene alineados los términos sexo biológico, género, deseo y práctica sexual, en una relación de tipo causal que solo admite la heterosexualidad como destino. De esta forma, los cuerpos y subjetividades que desafían las normas de inteligibilidad cultural que sostienen el binario normal-heterosexualidad/anormalhomosexualidad "se manifiestan únicamente como defectos en el desarrollo o imposibilidades lógicas desde el interior de ese campo" (Butler, 1990/2007, p. 73). Por tanto, terminan siendo expresiones de lo humano que solo se entienden en tanto patológicas y desviadas. Aproximarse al fenómeno de la patologización desde esta perspectiva expone el marco de inteligibilidad cultural que hace de fondo al debate actual sobre la patologización de las identidades trans y las posibilidades de cura de la homosexualidad. Y lo hace interrogando críticamente la supuesta objetividad con la que operan la psiquiatría y la psicología toda vez que asumen que sus posiciones respecto de la sexualidad y el género son neutras y no ideológicas, esto es, como si el sujeto normativo al que refieren se encontrara afuera de la historia y exento de los efectos de poder de otros marcadores de diferencia, como la clase, la raza, el género y la etnia, entre otros (Grzanka \& Miles, 2016).

Con la descripción precedente introduzco una aproximación posible al funcionamiento del poder patologizante, cuya historia, no obstante, es anterior a la creación de la APA y su Manual Diagnóstico y Estadístico de los Trastornos Mentales (DSM). Dicha historia se vincula a un legado colonial que no solo se refiere a las formas en que las categorías modernas de sexualidad y género se implantaron en las Américas, sino también a la invención del sistema sexo-género contemporáneo en el que se inscribe el par binario heterosexualidad/homosexualidad (Corrêa et al., 2014; Lugones, 2008; Wilkinson, 2013). Volviendo a Foucault (1976/2011), aquello que históricamente posibilitó la invención del homosexual como una especie distinta a la del sodomita del código penal y religioso se relacionaría con transformaciones políticas y epistemológicas acontecidas a comienzos del siglo XX, principalmente en Europa y los emergentes estadosnación latinoamericanos. Estas transformaciones no solo involucraron a las ciencias médicas y criminológicas de la época, sino también a una serie de actores, luchas sociales y formaciones discursivas que han incidido sobre las maneras en que se entiende actualmente la orientación sexual. Es así como algunos autores (Stein, 1999; Waidzunas, 2015) han sugerido comprender dichas transformaciones como un proceso en el que diversas formas de definir y medir la homosexualidad entran en conflicto, destacando, a modo general, un desplazamiento desde una definición basada en la conducta sexual a otra de tipo identitaria, en la que las referencias a la inmutabilidad y/o fluidez de la atracción sexual son disputadas.

\section{Los Dos Tiempos de la Despatologización: Normalizar y Repatologizar}

Estos movimientos en el plano de las definiciones no solo impactaron sobre el tipo de tratamientos clínicos disponibles, sino también sobre las formas en que las personas comenzaron a relacionarse con su propia sexualidad. Cuando la homosexualidad fue oficialmente clasificada como un trastorno mental por la APA en 1952 y reclasificada en 1968 como desviación sexual, no solo se legitimó el supuesto clínico que hacía de la homosexualidad una patología susceptible de ser tratada. Junto con esto, se institucionalizó la heterosexualidad como la única expresión de la sexualidad humana no patológica, desde donde se evaluaba —y continúa evaluándose - al otro-homosexual como diferente (Cornejo, 2011; Wilkinson, 2013).

La línea epistemológico-técnica del modelo de enfermedad/comportamiento que se articuló a partir de este giro patologizante (ver Drescher 2002, 2010), siguió una interpretación particular de la teoría del 
desarrollo psicosexual freudiano que fue muy influyente en el círculo psiquiátrico estadounidense a través del trabajo de los psicoanalistas Sandor Rado, Irving Bieber y Charles Socarides. Sus ideas han influido también la práctica profesional de analistas y psiquiatras en Latinoamérica, fortaleciendo conceptualmente muchos de los supuestos que inspiran actualmente a las principales intervenciones reparativas y/o de acompañamiento de la homosexualidad, principalmente masculina (Ojeda Güemes, 2015; Wilkinson, 2013). A modo general, dichos supuestos podrían resumirse de la siguiente manera: (a) la homosexualidad sería el síntoma de un desorden afectivo que da cuenta de una desviación o estancamiento en el desarrollo psicosexual normal; (b) dicha desviación y/o inmadurez en el desarrollo se explicaría por factores de tipo traumáticos acontecidos en la infancia o como consecuencia de una falla en las funciones parentales; (c) la homosexualidad sería una respuesta adaptativa ante la ausencia de modelos de identificación masculinos o femeninos y (d) el tratamiento sería, entonces, uno de tipo preventivo y/o reparativo del daño percibido hacia la identidad masculina o femenina (Bieber et al., 1962, citado en Bayer, 1987; Cohen, 2000/2012; Ferrer, 2007).

La transición desde un modelo patologizante a otro de tipo inclusivo de las sexualidades no normativas coincidió con la despatologización de la homosexualidad en 1973 y el desarrollo de discursos psicoterapéuticos afirmativos que lucharon por llevar a la homosexualidad de vuelta a la normalidad (Waidzunas, 2015). Una de las consecuencias de los procesos de lucha y politización del debate en torno a la patologización de la sexualidad lésbica, bisexual y gay al interior de la APA, fue la emergencia de una nueva ontología de la sexualidad que reforzó el carácter universal e inmutable de la homosexualidad en tanto variable natural de la sexualidad humana (Grzanka \& Miles, 2016). Esto último habría contribuido a que progresivamente las sociedades occidentales fueran modificando sus actitudes hacia la homosexualidad, incidiendo, de paso, en la despenalización socio-legal de la sodomía en varios países latinoamericanos durante la segunda mitad del siglo XX.

Una vez desclasificada la homosexualidad como un desorden psiquiátrico, el modelo de enfermedad/comportamiento fue gradualmente perdiendo su influencia en las corrientes teóricas dominantes de la salud mental (Drescher 2002, 2010). Sin embargo, en forma paralela, comenzó a articularse un nuevo frente opositor al interior de la APA, liderado principalmente por Socarides y el psicólogo estadounidense Joseph Nicolosi, quienes insistieron en restituir el carácter patológico de la homosexualidad. Para ello, desarrollaron una estrategia discursiva distinta a la utilizada en la época previa a 1973, que consistió en desplazar el objeto del debate desde la identidad gay hacia la conducta sexual y los estilos de vida. A partir de este giro repatologizante, la homosexualidad se comprendió como un comportamiento aprendido, no una identidad fija, y, como tal, podía ser modificada a través de la psicoterapia y/o la sanación espiritual (Drescher, 2002). Lo interesante de este movimiento es que la misma despatologización habría habilitado la posibilidad de cura e investigación clínica en torno a sus causas. Tal como señaló uno de los psiquiatras que apoyó su desclasificación, Robert Spitzer, una de las razones que habría permitido despatologizar la homosexualidad fue dejar en su reemplazo una categoría diagnóstica nueva, la de perturbación en la orientación sexual. Desde su visión, esta categoría permitía que la necesidad de tratamiento de quienes experimentaban cierto grado de malestar en relación a su atracción sexual homosexual pudiese seguir siendo reconocida; de lo contrario, "no había forma de que la homosexualidad fuera eliminada por completo del DSM-II" (Spitzer, p. 102, citado en Drescher, 2003).

\section{El Homosexual Sanado y el Impulso Reparativo}

La invención del dispositivo reparativo y su diseminación al interior de la psicología y los denominados movimientos exgay en Estados Unidos y Latinoamérica, surgió mayoritariamente como respuesta a la despatologización y legitimación progresiva de la homosexualidad en los campos de la salud mental, las políticas públicas, los derechos humanos y el activismo LGBTI (Wilkinson, 2013; Waidzunas, 2015). Según Nicolosi (1997), la despatologización de la homosexualidad excluía de la oferta de tratamiento clínico a un grupo de personas, en su mayoría hombres, que sí deseaban curarse de la AMS no deseada. A su juicio, estas personas no son gais, ya que en su experiencia no estaría la politización de su identidad ni el deseo de identificarse con un determinado estilo de vida que asocian con la promiscuidad, las conductas de riesgo y lo que algunos terapeutas han descrito como cultura de muerte (ver van den Aardweg, 1985/2005). A ellos más bien los motiva el deseo de cambiar e intervenir sobre todos aquellos aspectos de su vida que interfieren con el desarrollo de su potencial heterosexual.

En su libro Terapia Reparativa de la Homosexualidad Masculina: Un Nuevo Enfoque Clínico, Nicolosi (1997) presenta los fundamentos teóricos del dispositivo reparativo, señalando que la homosexualidad se explicaría por la existencia de un impulso que busca reparar un déficit en la identidad masculina que, en sí mismo, se entiende como traumático. El término reparativo proviene de la teoría sobre la homosexualidad 
desarrollada por la psicóloga y teóloga británica Elizabeth Moberly (1983), quien caracterizó la atracción erótica por el mismo sexo como un impulso de reparación. El componente traumático de esta experiencia es central dentro del modelo explicativo: la causa principal de la homosexualidad no sería la ausencia de un padre/madre, sino más bien la puesta en acto de un mecanismo de desapego defensivo que se activaría como defensa frente al rechazo - real o percibido - que el niño o la niña experimenta por parte de su figura paterna/materna (Moberly, 1983). En este contexto, los comportamientos homosexuales, vale decir, el acto sexual homosexual, son interpretados como expresiones de "un intento inconsciente de 'auto-reparar' sentimientos de inferioridad masculina" (Nicolosi, s.f., p. 3) y de satisfacer necesidades de intimidad emocional de tipo no sexual con personas de su mismo sexo.

El modelo de Nicolosi fue popularizado a través de la obra de Richard Cohen (2000/2012), quien, a su vez, desarrolló un modelo de recuperación basado en su propio itinerario de curación como exgay en su libro Comprender y Sanar la Homosexualidad. Este material se ha difundido activamente a través de conferencias, talleres y jornadas de capacitación en Estados Unidos, España, Chile, México y Uganda, entre otros países, documentándose, incluso, alianzas con sectores conservadores del catolicismo y el cristianismo pentecostal (Wilkinson, 2013). A diferencia de la propuesta de tratamiento de Nicolosi, la de Cohen es más flexible e integrativa y no solo está dirigida a profesionales del área de la salud mental, sino también a educadores, sacerdotes, religiosas y pastores. Esto último refuerza lo que otras investigaciones han señalado en relación a los vínculos entre psicología y religión para el caso del dispositivo reparativo (Morán \& Vaggione, 2012), el cual opera en la intersección de la práctica religiosa o espiritual y la práctica secular de la psicoterapia (Mikulak, 2018), recibiendo aportes de perspectivas afines a dichos vínculos, tales como la psicología tomista y el personalismo ontológico.

Dada su influencia, particularmente en Chile, describiré algunos de los aspectos más relevantes del modelo de Cohen (2000/2012) que permitan comprender cómo funciona la terapia reparativa en la práctica. En términos terapéuticos, la eficacia del modelo depende de al menos dos condiciones, a saber, la motivación y el deseo profundo de cambiar. Además de ello, y como condición de entrada al proceso curativo, se le pide al paciente que interrumpa su comportamiento sexual con personas de su mismo sexo y que termine con sus amistades en el mundo gay, adoptando un principio técnico similar al que utilizan las terapias de adicciones y el movimiento de los 12 pasos (Cohen, 2000/2012; Wilkinson, 2013). Sin estas condiciones, el cambio es virtualmente imposible. En términos descriptivos, su modelo de trabajo especifica un plan de tratamiento terapéutico inspirado en un paradigma de desarrollo lineal que divide en cuatro etapas, cada una de ellas con sus tareas y terapéutica específica: terapia de la conducta, cognitiva y psicodinámica. Estas, a su vez, incluyen una serie de técnicas que se utilizan con el fin de obtener los objetivos de cada una de las etapas, destacando, entre otras, la bioenergética, el psicodrama, la técnica de los abrazos y de reeducación del comportamiento, los juegos de rol, la psicoeducación, masajes terapéuticos y ejercicios físicos. Cohen (2000/2012) recomienda que durante las tres primeras etapas del proceso se trabaje con un terapeuta o consejero del mismo sexo que el paciente y añade que la decisión de iniciar este trayecto "lleva su tiempo, paciencia y un continuo esfuerzo" (p. 107). En relación a la noción de cambio terapéutico, tanto Cohen como Nicolosi ejemplifican el proceso de cura con el camino que realizan los adictos, señalando que aquello que se interrumpe y modifica es la conducta, no así la condición. La observación es confirmada también por los testimonios de exgais y la reivindicación que hacen de su propio proceso, ejemplificándolo, muchas veces, con el modo en que se entiende el cambio en las personas que son alcohólicas y que se encuentran en rehabilitación. Tal como advierte Nicolosi (1997), "el alcohólico nunca estará completamente curado de su deseo de beber, pero un tratamiento satisfactorio le ofrecerá una vía efectiva de lidiar con una condición que le acompañará de por vida. (p. 106).

De acuerdo con el análisis de Wilkinson (2013), la figura del homosexual sanado o del exgay es una de las invenciones del dispositivo y parte fundamental del proyecto político que subyace a las prácticas reparativas, las cuales buscan contrarrestar la legitimidad social y política de las identidades gais contemporáneas en su homologación con la heterosexualidad. El testimonio y experiencia del exgay sería evidencia de que el cambio es posible y que sería viable reivindicar una nueva tipología del sujeto homosexual que no se comprende únicamente desde la inmutabilidad, irreversibilidad y no elección de su atracción homoerótica (Wilkinson, 2013).

\section{Ideas que Viajan: La Dimensión Transnacional del Discurso Reparativo y el Caso Chileno}

La circulación del término reparativo al interior de la psiquiatría y psicología en Estados Unidos y Latinoamérica ofreció una explicación causal a la homosexualidad que habilitó una serie de ofertas de ayuda para pacientes altamente motivados que deseaban cambiar su atracción sexual homoerótica. Hoy en día la 
mayoría de las y los profesionales de la salud que adhieren a los supuestos del cambio de la homosexualidad en Chile se refieren a lo que ofrecen como acompañamientos para personas que sufren de AMS no deseadas o de esfuerzos por cambiar la orientación sexual, dejando afuera estratégicamente los términos reparativo, reorientación y/o conversión. Algo similar ocurre con las formas de nominación de la homosexualidad, principalmente masculina, donde el lenguaje científico-médico se reemplaza y/o confunde con otro de tipo religioso: algunos terapeutas y organizaciones señalan que la homosexualidad no existe (ver Schroeder \& Shidlo, 2002) y prefieren hablar de tendencias o inclinaciones homosexuales, proyección hacia el mismo sexo, heridas emocionales o AMS (Es Posible la Esperanza, 2013; van den Aardweg, 1985/2005). Asimismo, no todas/os las y los profesionales se identifican como psicoterapeutas: algunos trabajan como coaches, acompañantes y/o consejeros (Lorenzo, 2014; Ríos, 2014, Octubre 3), utilizando técnicas de apoyo de la psicología, el acompañamiento espiritual y el coaching, de manera intercambiable y sin límites claros.

Si bien gran parte de la producción de conocimientos en torno a la cura de la homosexualidad se generó mayoritariamente en Estados Unidos en un momento histórico-político específico, muchas de las ideas que han ido gestándose en dicha locación han sido importadas y modificadas en Latinoamérica en respuesta a situaciones bastante similares. El discurso reparativo habría hecho sentido en América Latina en tanto reacción frente a la creciente legitimidad del activismo LGBTI y la despenalización socio-legal de la homosexualidad en muchos de los países de la región. Esto coincidiría históricamente con el restablecimiento parcial de las democracias en contextos de post dictadura y la asimilación progresiva de la normativa institucional no patologizante de la APA y la OMS en las políticas locales de salud y derechos humanos (Barrientos, 2015; Cornejo, 2011; Pecheny \& de la Dehesa, 2014; Wilkinson, 2013).

Lo que Wilkinson (2013) denomina transnacionalización de los discursos y prácticas reparativas en su análisis de la cura gay en Ecuador, se refiere, por un lado, a las condiciones de posibilidad histórico-políticas que hacen que determinados contextos geográficos estén más disponibles a la recepción y adaptación de ciertas ideas patologizantes. Y, por otro lado, lo transnacional se refiere también a la organización del conocimiento en sí y las redes de transmisión que se articulan en torno a un determinado proyecto político común, en este caso, la defensa de la heterosexualidad como única expresión de la sexualidad normal (Ojeda, 2019, Abril 13). A nivel global, la legitimación y normalización de las identidades gais a través del activismo LGBTI no solo ha incidido en la regulación normativa de la subjetividad sexual contemporánea mediante políticas liberales de tolerancia, inclusión y diversidad, ampliamente discutidas por la crítica queer y feminista (Sabsay, 2012; ver también Galaz Valderrama, Sepúlveda Galeas, Poblete Melis, Troncoso Pérez \& Morrison Jara, 2018). Este tipo de avances en materia de derechos sexuales también ha motivado la creación de otro frente de resistencia que se organiza en torno a la figura del exgay, el cual se erige como alternativa frente a la hegemonía de las identidades gais y lésbicas y los discursos afirmativos de las sexualidades no heterosexuales (Wilkinson, 2013). En torno a la figura del exgay circulan nuevas formas de homofobia amparadas en discursos de protección de la libertad de expresión y de enseñanza, que operan con relativa legitimidad y en los límites de lo que se considera como propio de una racionalidad secular, en contraposición a otra de tipo religiosa. Es así como las prácticas reparativas se promueven a través de ministerios cristianos y organizaciones profesionales vinculadas a la psicología, bioética, pediatría y educación, muchas de ellas de inspiración abiertamente religiosa y con vínculos en universidades, centros de estudios, organizaciones no gubernamentales (ONGs) y partidos políticos. A modo de ejemplo, y siguiendo la descripción que hace Wilkinson (2013), en Latinoamérica destacan los ministerios religiosos de tradición católica y evangélica pentecostal, tales como Courage Latino, el Grupo Juan Pablo II (responsables del consultorio virtual www.esposiblelaesperanza.com) y Exodus Latinoamérica, con sus filiales en Ecuador, Camino de Salida, en Chile, Nueva Esperanza, y en Argentina, Nueva Condición. También destacan diversas instituciones de salud, activismo político y acompañamiento religioso, como el Centro Areté en Perú, la ONG ISFEM y la Fundación Restauración en Chile, entre otras (Ojeda, 2019, Abril 13; Ojeda Güemes, 2015).

Si miramos el caso de la Fundación Restauración, me detendré en el rol que han tenido dos de sus integrantes, la psicóloga Marcela Ferrer y el médico pediatra Christian Schnake, ambos titulados con un grado de Magíster en Bioética por el Centro de Bioética de la Facultad de Medicina de la Pontificia Universidad Católica de Chile. Si bien Ferrer y Schnake han defendido públicamente sus posturas en distintos medios (prensa, charlas, artículos de difusión académica), no son las únicas personas que ofrecen este tipo de acompañamientos para tratar la AMS ni tampoco los únicos que difunden sus creencias en torno al cambio de la homosexualidad. Además de sus publicaciones, están los artículos recopilados en el libro Sobre la Homosexualidad que editaron académicas/os de la Universidad de los Andes el año 2007, tras la visita del psicólogo holandés Gerard van den Aardweg el año 2004 en el marco de un seminario que abordó las 
posibilidades de cura de la homosexualidad (Ojeda, 2019, Abril 13). El año 2008, el Centro de Bioética de la Universidad Católica editó un libro sobre la homosexualidad desde una perspectiva antropológico-cristiana, a cargo del sacerdote Fernando Chomalí, en coautoría con Ferrer, Schnake, Alejandra Carrasco y Paulina Johnson, el cual profundiza en las supuestas causas de la homosexualidad y discute, también, sus posibilidades de cura. Años después, en octubre de 2012, la ONG provida ISFEM organizó un seminario en la casa central de la Universidad Católica de Santiago que reunió, entre otros, a las y los principales exponentes de las prácticas reparativas en Latinoamérica y contó con el testimonio de un activista exgay de las Islas Canarias. Este seminario puso en escena las alianzas que existen entre agrupaciones provida y sectores conservadores de la academia en Chile, que buscan constituirse como un frente de resistencia ante la pérdida de los espacios de privilegio con que circulaban sus ideas en torno a la patologización de las diferencias (Ojeda, 2019, Abril 13).

De acuerdo al análisis realizado por el investigador Juan Rolando Cornejo (2011) en torno a la medicalización de la homosexualidad en Chile, el paradigma patologizante continuó presente en los campos de la psiquiatría y la bioética durante la década de los 90 y principios de los 2000, prolongándose incluso hasta el día de hoy. De acuerdo al autor, este tipo de discursos se habría articulado en respuesta a transformaciones socioculturales y políticas vinculadas, en su mayoría, a la pérdida de hegemonía discursiva del paradigma medicalizador por parte de médicos, políticos y autoridades religiosas. A lo anterior se sumarían otros dos elementos que, a mi juicio, explican parcialmente la relativa legitimidad que tienen actualmente los discursos reparativos en ciertos círculos académicos y político-religiosos. El primero de ellos estaría conectado con la creciente mercantilización de la psicoterapia y las influencias del neoliberalismo en las políticas de salud y de sexualidad contemporáneas. Esto habría influido en que la discusión se desarrolle principalmente en torno a los criterios de eficacia de las prácticas reparativas y la invocación a la libertad de elección, esfuerzo y motivación como argumentos para legitimar o prohibir su circulación. El segundo elemento interroga críticamente el proyecto político y religioso que defienden las y los partidarios de la cura gay en Chile, evidenciando el poder heteronormativo del discurso patologizante y las paradojas en torno al carácter aprendido de la heterosexualidad.

\section{Obligados a Elegir}

En Chile, la crítica en torno a las tecnologías del cambio de la identidad sexual (Cohen, 2000/2012) se ha centrado casi exclusivamente en dos aspectos, a saber, en un cuestionamiento de tipo ético contra la no efectividad y el fraude asociado a su promesa de cambio, y en una disputa en torno a la evidencia que documenta los riesgos asociados a este tipo de prácticas y el carácter no patológico de las identidades trans y no heterosexuales. Por otro lado, pese al consenso y a la literatura disponible respecto de estos puntos, los testimonios de exgais y la supuesta demanda de atención clínica por parte de personas con AMS operan simultáneamente como evidencia en contra del pretendido origen genético de la orientación sexual y como criterio de verificación del dispositivo reparativo (Chomalí, 2008; Zegers, Larraín \& Bustamante, 2007). Este último aspecto ha instalado un cuestionamiento a los mecanismos de regulación y las facultades de fiscalización que emergen de los propios colegios profesionales, los cuales, en el caso de la psicología, no tienen competencia sobre psicólogas/os y terapeutas no colegiados (Colegio de Psicólogos de Chile, 2015a).

Uno de los argumentos más movilizados para apoyar este tipo de iniciativas es el de la libertad. En Chile, quienes ofrecen este tipo de alternativas lo hacen señalando que sus pacientes eligen libremente participar del proceso. En relación a la oferta terapéutica, Ferrer (2007) reconoce que "no se puede siempre garantizar el resultado" (p. 100) y uno de los aspectos que advierte desde un principio "es que el hecho de hacer un camino terapéutico en psicología y psiquiatría (...) no es una garantía de que la persona va a llegar a obtener una atracción heterosexual" (Ríos, 2014, Octubre 3, párr. 10). Es más, Ferrer aclara que ella "no habl[a] de curar la homosexualidad" (Ortiz, 2017, Diciembre 9, párr. 32) ya que "la atracción homosexual no se quita, sino que se ordena" (párr. 33). Por lo tanto, es la persona quien debe "aprender cómo y qué [quiere] hacer con esa atracción", lo cual "va a depender de la fuerza que [tenga] y de las elecciones que [vaya] haciendo" (párr. 33).

Para efectos del análisis, me interesa centrarme en las condiciones en que se negocian las expectativas y los criterios de entrada al proceso terapéutico y en las formas en que la práctica terapéutica produce una noción particular de libertad de elección, esfuerzo y motivación en tanto capacidades de los individuos y no como productos de una determinada lógica de acompañamiento (Mol, 2006/2008). Esta distinción es clave. Según Ferrer (Ortiz, 2017, Diciembre 9), su propuesta se justifica en el entendido de que cubre una necesidad, tal como lo justificó en su momento Nicolosi al reivindicar las necesidades de los denominados homosexuales 
no-gay. Para que la oferta de ayuda sea efectiva, la persona debe estar altamente motivada y creer en la posibilidad del cambio (Cohen, 2000/2012). Es decir, la supuesta efectividad de la cura depende de variables de tipo individuales y no necesariamente de una técnica terapéutica. Si la persona elige libremente participar en este tipo de terapias, ella será quien responda por sus consecuencias, especialmente en términos del logro de la atracción heterosexual y el control de su tendencia homoerótica. Y esto último es particularmente problemático en el contexto económico y político en que se entiende la salud mental y la oferta terapéutica en Chile.

Diversos autores han interrogado los vínculos entre psicoterapia, mercado y neoliberalismo en Chile, discutiendo críticamente los problemas asociados a comprender el cuidado y la salud como bienes de consumo y de responsabilidad individual (Ferrer, 2013; Radiszcz, 2009; ver también Aceituno Morales, Miranda Hiriart \& Jiménez Molina, 2012; Han, 2012). Siguiendo la línea de análisis de la socióloga chilena Marcela Ferrer Lues (2013, Septiembre-Octubre), esto último ha repercutido en la articulación de una demanda de acompañamiento y trabajo clínico que hace equivalentes la salud con la consecución de estilos de vida saludables, que para el caso de la terapia reparativa se traduce en la expectativa de obtener un estilo de vida heterosexual. En sus semejanzas con las lógicas de funcionamiento de la psicoterapia basada en la evidencia, el dispositivo reparativo promueve el ajuste del individuo a un ideal normativo que tiende a su homogeneización (Radiszcz, 2009) y que, en última instancia, requiere del convencimiento del paciente -y su esfuerzo individual - para que el cambio sea posible (Cohen, 2000/2012; Wilkinson, 2013). De esta forma, el dispositivo transforma a la psicoterapia "en un campo de administración de la vida" que disuelve la singularidad "para someterla a una uniformidad poblacional posible de gobernar" (Radiszcz, 2009, p. 121).

En relación al objeto de tratamiento, y parafraseando a la teórica queer Eve Kosofski Sedgwick (1992/1993), la intervención terapéutica reparativa actuaría directamente sobre cualidades de la voluntad individual que terminan definiendo a la AMS no tanto en referencia a su naturaleza desviada o antinatura, sino como un déficit en las habilidades de autocontrol y disciplina. Estas, a su vez, pasan a ser los objetos del acompañamiento y/o de la intervención clínica, en tanto interfieren con la capacidad de las personas para elegir libremente la salud, en este caso, la heterosexualidad. Lo patológico, desde esta perspectiva, residiría en la estructura de una voluntad que se concibe como insuficientemente libre, por tanto, la afirmación de dicha voluntad pasa a ser el objeto de apoyo y tratamiento clínico (Sedgwick, 1992/1993). En este contexto, la misma libertad que se exige como requisito de entrada al proceso terapéutico se vuelve problemática durante el transcurso del acompañamiento, no solo porque supone que todos los sujetos son igualmente libres para decidir y negociar los términos de la relación terapéutica (Mol, 2006/2008), sino también porque dicho acompañamiento instala una exigencia sobre la voluntad que, en muchos casos, impone una decisión forzada entre posiciones que son por definición excluyentes (Ojeda Güemes, 2015). Para intervenir sobre la atracción sexual no deseada, por ejemplo, el individuo debe elegir entre comportarse o no como homosexual y el dispositivo lo fuerza a ello a través de metodologías voluntaristas que producen sus propios quiebres, especialmente cuando el individuo fracasa en la consecución de sus objetivos o recae en antiguas prácticas sexuales proscritas por la indicación terapéutica (Schroeder \& Shidlo, 2002). En dichos casos, lo que aparece es la experiencia de la culpa en su dimensión patológica, una adhesión obsesiva a ideales sociales de dominio de sí, disciplina mental e interiorización del control de las pulsiones sexuales y del sentido de la responsabilidad llevada a sus últimas consecuencias (Castel, 2014). Entre ellos destaca la ideación y tendencia suicida, el consumo problemático de sustancias y el desarrollo de cuadros ansiosos y depresivos, lo cual se encuentra ampliamente documentado en guías clínicas, reportes y testimonios que advierten sobre los daños y vulneraciones a los derechos humanos de este tipo de prácticas (American Psychological Association, 2009; Amnistía Internacional, 2001; Colegio de Psicólogos de Chile, 2015a, 2015b; Organización Panamericana de la Salud, 2012; Waidzunas, 2015; Wilkinson, 2013).

Según la lógica descrita, si la persona no cambia es porque no se esforzó lo suficiente, porque nunca estuvo convencida o porque no le creyó al terapeuta ni a sí misma. Paradójicamente, es el exceso de normalidad lo que deviene en un objeto de sufrimiento para el individuo (Castel, 2014). La misma libertad que aparece como garante del proceso terapéutico produce también sus propios síntomas y malestares, aquello que Sedgwick (1992/1993) describe de manera muy elocuente como patologías de la voluntad en el contexto de la cura y tratamiento de las adicciones, lo cual es tremendamente ilustrativo para el caso de las prácticas reparativas, puesto que la AMS es descrita como un comportamiento de tipo adictivo (Nicolosi, 1997; van den Aardweg, 1985/2005). 


\section{Aprendiendo a Ser Heterosexuales}

La lógica neoliberal o de libre elección que subyace a este tipo de prácticas (ver Mikulak, 2018) va de la mano con una argumentación de tipo política y moral que promueve la heterosexualidad como principio normativo. Es aquí donde identifico una contradicción, pues, si bien quienes defienden el dispositivo reparativo movilizan la libertad como argumento para legitimar sus demandas y ocultar sus aspiraciones ideológicas, las prácticas reparativas no son neutrales y, por el contrario, promueven un estilo de vida particular.

Una mirada crítica a la discusión en torno a la eficacia del cambio apunta a la dimensión moral y normativa del dispositivo reparativo en sus esfuerzos por definir objetivamente sus posibles resultados. Aun cuando existan diferencias respecto de cómo medir la meta terapéutica (ver Waidzunas, 2015), la mayoría de los modelos reparativos y de acompañamiento operan bajo el supuesto de que todo homosexual es un heterosexual latente (Bieber et al., 1962, citado en Drescher, 1998) y es esa también la expectativa que moviliza una demanda particular de cura, que en la mayoría de los casos se desarrolla de manera bastante homogénea, independientemente de sus efectos en el mediano o largo plazo y del género de quien consulta.

Tal como sugiere el psicoanalista Esteban Radiszcz (2009) para el caso de la terapia basada en la evidencia, la definición de metas y resultados tiende a producir un ideal de normalidad que, en último término, solo podría informar "cuánto ha servido un tratamiento para corregir una desviación subjetiva" (p. 121), en este caso, respecto de la atracción heterosexual. El componente ideológico de este tipo de tratamientos queda en evidencia cuando la pregunta respecto de los efectos de promover la creencia de que el cambio es posible considera, también, el régimen de gubernamentalidad en el que se sostiene el dispositivo completo (Radiszcz, 2009), el cual alimenta los imaginarios sociales de enfermedad que siguen acechando a las personas gais, lesbianas, bisexuales y trans; aquello que la escritora feminista Adrienne Rich (1986/2001) describió como heterosexualidad obligatoria en sus esfuerzos por apuntar al carácter político e institucional de la heterosexualidad más allá de su referencia identitaria.

El análisis del itinerario de sanación de las propuestas de acompañamiento de Cohen y Ferrer (Ojeda Güemes, 2015) y de algunos de los psicólogos cristianos analizados por Wilkinson (2013), revela que la anhelada identidad heterosexual que muchos buscan obtener no es más que el producto de un programa de rectificación pedagógica que requiere del esfuerzo individual y de una serie de apoyos que ayuden a mantener la decisión. Su currículum garantiza el aprendizaje de estrategias y habilidades de coping que obtienen su legitimidad a partir del testimonio de exgais que confirman que son portadores creíbles de los atributos que hacen que cada paciente pueda "pasar" como un verdadero heterosexual. En este contexto, el yo del individuo se comprende como un complejo de hábitos adquiridos a través de un proceso de asimilación imitativa que se refuerza constantemente con distintos tipos de técnicas, entre ellas, la biblioterapia (lectura de libros, artículos y testimonios acerca de las causas de la homosexualidad), la participación en grupos de pares y la relación terapéutica (Cohen, 2000/2012). Estas tres técnicas terminan por homogeneizar los referentes y las formas de ser un sujeto sexuado, haciendo de la experiencia singular una copia más de lo mismo.

En este contexto, los terapeutas y acompañantes ocupan el lugar de técnicos de la conducta "por la vía de una técnica estandarizada —e, incluso, manualizada—" (Radiszcz, 2009, p. 122) que busca restablecer la norma y procurar la gestión adecuada de las tendencias homoeróticas. La intervención terapéutica confirma en el relato de quienes consultan lo que la teoría - y las creencias de los propios profesionales- tiene previamente tipificada, haciendo coincidir de manera violenta el lugar de enunciación de los pacientes con un sentido que totaliza sus experiencias y que irrumpe sin posibilidades de ser desmentido (Ojeda Güemes, 2015). El hecho de que Cohen (2000/2012) insista en el poder curativo del vínculo con terapeutas y/o mentores en tanto representantes del ideal de masculinidad o feminidad al que aspiran las y los pacientes, da cuenta de los riesgos asociados a una intervención clínica que favorece un tipo de identificación reñida con la ética y que apuesta a que la heterosexualidad podría trasmitirse por contacto (ver nota escrita por Boodman, 2005, Agosto 16, en The Washington Post, a propósito de la expulsión permanente de Cohen de la Asociación Norteamericana de Consejería). Esto último ha sido ampliamente debatido en artículos que han evaluado la efectividad del dispositivo reparativo, reportándose, en muchos casos, vulneraciones en el ámbito de la relación terapéutica, toda vez que terapeutas y/o consejeras/os han asumido el rol del padre o la madre del paciente con el fin de reparar los supuestos traumas asociados a sus heridas emocionales (Schroeder \& Shidlo, 2002), muchas veces empujados por aquello que Cohen (2000/2012) denomina como el poder curativo del tacto y los abrazos. 
Con todo, los testimonios de exgais, en conjunto con algunas de las técnicas terapéuticas que he descrito, refuerzan la idea de que la heterosexualidad, en cualquiera de sus versiones (en tanto identidad, atracción o estilo de vida), sería más bien una expresión de la sexualidad que se comprende como construida y aprendida, antes que natural, verdadera u original (Ojeda Güemes, 2015). Al defender esto último, el dispositivo completo se sostiene en la convicción de que existe una verdad en estado potencial que habría que descubrir. Por tanto, la teoría que conceptualiza a la homosexualidad como un síntoma o como un trastorno del desarrollo no solo cree en la existencia de un tratamiento curativo y/o preventivo, sino también instituye un principio de inteligibilidad política y cultural que apuesta por un fundamento trascendente que habría que restituir. Y es esta lógica restitutoria la que inscribe al dispositivo reparativo dentro de lógicas de regulación heteronormativas que, además de reivindicar el estatuto natural de la heterosexualidad y el binarismo de género, favorecen la proliferación de discursos patologizantes que legitiman la violencia homo, lesbo y transfóbica (Ojeda, 2019, Abril 13).

Lo interesante y a su vez paradójico es que, pese a su poder, es el mismo dispositivo el que revela el carácter ficticio y contingente de la norma heterosexual, la cual necesita de la homosexualidad en su vertiente patológica para conservar su eficacia como régimen político. En cuanto tal, esta terapéutica pareciera estar más comprometida con una lógica de regulación de la sexualidad masculina al reproducir un orden de género particular que invisibiliza la sexualidad de las mujeres, particularmente lesbianas y bisexuales (Mikulak, 2018). Esto último resulta especialmente problemático, puesto que el modelo reparativo supone que la homosexualidad femenina se explicaría casi de la misma forma como se entiende la homosexualidad masculina. Desde dicha lógica, las vicisitudes del deseo de mujeres lesbianas o bisexuales se explica por su orientación casi exclusiva al mundo de los cuidados, por ende, desexualizado y en referencia a la norma masculina, agregando, incluso, que la supuesta fluidez de la sexualidad femenina sería evidencia suficiente contra el argumento de que la homosexualidad estaría biológicamente determinada (Diamond, 2009; Waidzunas, 2015). Las mujeres, en este modelo, aparecen como objetos de intercambio para regular la sexualidad masculina y preservar el orden reproductivo natural (Rich, 1986/2001), especialmente cuando son referidas en los testimonios de exgais como indispensables para el logro de la atracción heterosexual masculina en tanto parejas sexuales, esposas y/o madres -ver testimonios recopilados en textos académicos, libros de casos y reportajes periodísticos en Nicolosi (1993/2009), Prieto Damm (2017, Febrero 17), Solari (2010, Octubre 22) y Wilkinson (2013).

\section{Conclusiones}

En este artículo propongo una lectura crítica del debate en torno a la denominada cura gay en Chile a través del análisis de una de sus manifestaciones más controversiales en el campo de la psicoterapia: las prácticas reparativas o de acompañamiento a personas que "sufren" de AMS no deseada. En su especificidad, me propuse interrogar críticamente algunas de las condiciones históricas, sociopolíticas y económicas que han posibilitado que este tipo de discursos gocen de cierta legitimidad en el presente, evaluando el dispositivo reparativo en dos dimensiones escasamente exploradas en la literatura especializada.

La primera de ellas se relaciona con las condiciones que son invocadas al momento de reivindicar la posibilidad del cambio de la homosexualidad, a saber, la motivación, el esfuerzo y la libertad individual. Estos tres elementos no solo son relevantes para el análisis de las prácticas reparativas, sino que son también principios técnicos que podemos encontrar en muchas otras psicoterapias. Esto último hace extensiva la crítica que he desarrollado en este artículo a la forma con que operan otras terapéuticas, las cuales podrían hacer de la demanda de cura y cambio de la AMS un objeto de trabajo clínico compatible con el ejercicio de la profesión. Tal como lo demuestro en este trabajo, resulta necesario pensar de qué formas la creciente mercantilización de la psicoterapia incide en las maneras en que se ofertan determinados estilos de vida e identidades, afectando, de paso, las formas en que se articula una demanda por la salud y la normalidad que generan sus propios quiebres sintomáticos y mecanismos de exclusión. La crítica, en este contexto, es a los supuestos que alimentan la creencia en el estatuto anormal, antinatura y patológico de la homosexualidad y las identidades trans, al mismo tiempo que a "la oferta de una 'solución' frente a cualquier demanda" (Ojeda Güemes \& Avaria, 2017, Febrero 20, párr. 10).

Una segunda dimensión que he abordado en este artículo recoge otro de los elementos de contexto que hacen que este tipo de discusiones siga teniendo relativa vigencia en Chile y Latinoamérica. Así como en sus orígenes, el discurso reparativo moviliza parte de las estrategias de resistencia de sectores de la población que ven amenazados sus privilegios ante la creciente visibilización de las luchas políticas feministas y de 
colectivos LGBTI. En este contexto, la defensa de la heterosexualidad en tanto régimen político y los esfuerzos por documentar los logros de la atracción heterosexual a través de los testimonios de activistas exgais forman parte del proyecto ideológico que reivindican los grupos que promueven la cura gay al interior de los espacios educativos, las iglesias y los partidos políticos. Si no prestamos atención a dichas formas de resistencia, así como a las nuevas alianzas que han ido gestándose entre organizaciones políticas, académicas y religiosas, arriesgamos la posibilidad de articular un marco analítico alternativo que desafíe dichos esfuerzos por convertirse en un nuevo sentido común en el ámbito de las libertades individuales y los derechos humanos (Corrêa, Paternotte \& Kuhar, 2018; Ojeda, 2019, Abril 13). Y esto no solo se logra exponiendo el sinsentido de muchas de sus demandas, sino también escuchando cuáles son los núcleos de temor y ansiedades a las que responden, y los elementos históricos, económicos y culturales a los que apelan como fuentes para legitimar sus discursos patologizantes y sus prácticas discriminatorias.

Con todo, mi artículo podría estimular el desarrollo de investigaciones empíricas que permitan comprender de mejor manera cómo funcionan las terapias y acompañamientos reparativos en Chile. Las ideas que he expuesto contribuyen a analizar dichos dispositivos no solo en términos del impacto sobre la salud física y mental de quienes las han experimentado, sino también sobre los factores ideológicos y políticos en los que se apoyan. Como ya vimos, muchas de las condiciones que hacen que este tipo de dispositivo sea efectivo en sus esfuerzos por posicionarse como alternativa de ayuda legítima, no son muy distintas de las formas en que operan otro tipo de terapéuticas y relaciones de ayuda. El desafío, entonces, es revisar lo propiamente terapéutico del dispositivo y disputar el sentido de los términos que utilizan, especialmente aquellos que aluden a campos disciplinares que han sido claros, al menos recientemente, en su condena contra la patologización de las diferencias. El uso instrumental de la psicología al servicio de un proyecto ideológico como el que he descrito da cuenta del uso abusivo de la psicoterapia para reproducir la violencia homo, lesbo y transfóbica, legitimando, con ello, la enseñanza de prácticas discriminatorias que atentan contra los derechos de las personas y su bienestar (Barrientos \& Cárdenas, 2013; Colegio de Psicólogos de Chile, 2015b).

\section{Referencias}

Aceituno Morales, R., Miranda Hiriart, G. \& Jiménez Molina, Á. (2012). Experiencias del desasosiego: salud mental y malestar en Chile. Anales de la Universidad de Chile, 3, 87-102. https://doi.org/10.5354/0717-8883.2012.21730

American Psychological Association (2009). Report of the American Psychological Association Task Force on appropriate therapeutic responses to sexual orientation. Washington, DC: Autor. Extraído de http://www.apa.org/pi/lgbt/resources/therapeutic-response.pdf

Amnistía Internacional (2001). Crímenes de odio, conspiración de silencio: tortura y malos tratos basados en la identidad sexual. London, Reino Unido: Autor. Extraído de https://www.amnesty.org/download/Documents/120000/act400162001es.pdf

Barrientos, J. \& Cárdenas, M. (2013). Homofobia y calidad de vida de gay y lesbianas: una mirada psicosocial. Psykhe, 22(1), 3-14. https://doi.org/10.7764/psykhe.22.1.553

Barrientos, J., Díaz, J. L., Gómez, F. \& Muñoz, F. (2012). Derechos, política, violencia y diversidad sexual: Segunda Encuesta Marcha de la Diversidad Sexual Santiago de Chile 2011. Antofagasta, Chile: Universidad Católica del Norte, Movimiento por la Diversidad Sexual. Extraído de http://www.clam.org.br/uploads/arquivo/Derechos,\%20politica,\%20violencia\%20y\%20diversidad\%20\%20segunda\%20encuesta\%20Santiago.pdf

Barrientos Delgado, J. (2015). Violencia homofóbica en América Latina y Chile. Santiago, Chile: Ediciones El Desconcierto.

Bayer, R. (1987). Homosexuality and American psychiatry: The politics of diagnosis. Princeton, NJ: Princeton University Press.

Boodman, S. G. (2005, Agosto 16). A conversion's therapists unusual oddisey. The Washington Post, Health. Extraído de http://www.washingtonpost.com/wp-dyn/content/article/2005/08/15/AR2005081501063.html??noredirect=on

Borba, R. (2017). Ex-centric textualities and rehearsed narratives at a gender identity clinic in Brazil: Challenging discursive colonization. Journal of Sociolinguistics, 21, 320-347. https://doi.org/10.1111/josl.12236

Butler, J. (1990/2007). El género en disputa: el feminismo y la subversión de la identidad (M. A. Muñoz, Trad.; Título original: Gender trouble: Feminism and the subversion of identity). Barcelona, España: Paidós.

Castel, P. -H. (2014). Vers une autre histoire de nous-mêmes, à l'ombre des obsessions-compulsions [Hacia otra historia de nosotros mismos, a la sombra de las obsesiones-compulsiones]. Extraído de http://pierrehenri.castel.free.fr/

Chomalí, F. (2008). Algunas consideraciones para el debate actual acerca de la homosexualidad: antecedentes científicos, antropológicos, éticos y jurídicos en torno a las personas y las relaciones homosexuales. Santiago, Chile: Pontificia Universidad Católica de Chile, Facultad de Medicina, Centro de Bioética.

Cohen, R. (2000/2012). Comprender y sanar la homosexualidad (J. A. Ullate, Trad.; Título original: Coming out straight: Understanding same-sex attraction). Madrid, España: Libros Libres.

Colegio de Psicólogos de Chile (2015a). Fundamentos técnicos: posición del Colegio de Psicólogos de Chile acerca de las terapias reparativas para curar la homosexualidad. Santiago, Chile: Autor. Extraído de http://colegiopsicologos.cl/wpcontent/uploads/2018/02/Referencias-Tecnicas-Terapias-Reparativas-Revision-final-04-Junio-2015.pdf

Colegio de Psicólogos de Chile (2015b). Comunicado público: posición del Colegio de Psicólogos de Chile A.G. sobre las terapias reparativas para curar la homosexualidad. Santiago, Chile: Autor. Extraído de http://terapiafamiliar.cl/nuevositio/wpcontent/uploads/2018/04/DeclaracionCol_Psic_Terapias_Reparativas.pdf 
Contardo, O. (2011). Raro: una historia gay de Chile. Santiago, Chile: Planeta.

Cornejo, J. R. (2011). Configuración de la homosexualidad medicalizada en Chile. Sexualidad, Salud y Sociedad, 9, $109-136$. https://doi.org/10.1590/S1984-64872011000400006

Corrêa, S. (2018). Ideología de género: rastreando sus orígenes y significados en la política de género actual. Rio de Janeiro, Brasil: Sexuality Policy Watch. Extraído del sitio Web de Sexuality Policy Watch: http://sxpolitics.org/es/ideologia-de-genero-rastreandosus-origenes-y-significados-en-la-politica-de-genero-actual/3858

Corrêa, S., Davis, B. R. \& Parker, R. (2014). Sexuality and globalities. En D. L Tolman \& L. M. Diamond (Eds.), APA handbook of sexuality and psychology. Volume 2: Contextual approaches (pp. 267-308). Washington, DC: American Psychological Association.

Corrêa, S., Paternotte, D. \& Kuhar, R. (2018). Europa, América Latina y la globalización de las campañas contra el género [en línea]. Democracia Abierta. Extraído de https://www.opendemocracy.net/democraciaabierta/sonia-correa-david-paternotte-romankuhar/europa-am-rica-latina-y-la-globalizaci-n

Diamond, L. M. (2009). Sexual fluidity: Understanding women's love and desire. London, Reino Unido: Harvard University Press.

Drescher, J. (1998). I'm your handyman: A history of reparative therapies. En A. Shidlo, M. Schroeder \& J. Drescher (Eds.), Sexual conversion therapy: Ethical, clinical, and research perspectives (pp. 5-24). New York, NY: Haworth Medical Press. https://doi.org/10.1300/J082v36n01_02

Drescher, J. (2002). Sexual conversion ("reparative") therapies: A history and update. En B. E. Jones \& M. J. Hill (Eds.), Mental health issues in lesbian, gay, bisexual, and transgender communities (pp. 71-91). Washington, DC: American Psychiatric Association.

Drescher, J. (2003). An interview with Robert L. Spitzer, MD. En J. Drescher \& J. P. Merlino (Eds.), American psychiatry and homosexuality: An oral history (pp. 95-112). Binghamton, NY: Harrington Park Press/Haworth Press.

Drescher, J. (2010). Queer diagnoses: Parallels and contrasts in the history of homosexuality, gender variance, and the diagnostic and statistical manual. Archives of Sexual Behavior, 39, 427-460. https://doi.org/10.1007/s10508-009-9531-5

Es Posible la Esperanza (2013). Es posible salir de la AMS (Atracción hacia personas del mismo sexo) [Video]. Extraído de https://www.youtube.com/watch?v=qRu62DafDeA

Fassin, É. (2006/2012). La democracia sexual y el choque de civilizaciones (M. Belgrano Rawson, Trad.; Título original: La démocratie sexuelle et le conflit des civilisations). Mora: Revista del Instituto Interdisciplinario de Estudios de Género, 18, 5-10. Extraído de http://revistascientificas.filo.uba.ar/index.php/mora/article/view/322

Ferrer, M. M. (2007). Percepción infantil de no ser aceptado como un factor predisponente a la homosexualidad (Tesis de Magíster, Pontificia Universidad Católica de Chile, Santiago, Chile). Extraído de https://www.bioeticacs.org/iceb/investigacion/4_Ferrer_Marcela_Prevencion_homosexualidad.pdf

Ferrer, M. (2013, Septiembre-Octubre). ¿La salud como responsabilidad individual? Análisis del concepto de salud en los programas de gobierno de la Concertación en Chile. Ponencia presentada en el XXIX Congreso de la Asociación Latinoamericana de Sociología, Santiago, Chile. Extraído de https://documentop.com/universidad-de-chile-acta-cientifica-congreso-alas-chile_5a048cf51723dda8d191751d.html

Figari, C. (2012). La invención de la sexualidad: el homosexual en la medicina argentina (1880-1930). En D. Jones, C. Figari y \& S. Barrón López (Coords.), La producción de la sexualidad: políticas y regulaciones sexuales en Argentina (pp. 19-44). Buenos Aires, Argentina: Biblos.

Foucault, M. (1976/2011). Historia de la sexualidad: 1-La voluntad de saber (3 ${ }^{\mathrm{a}}$ ed.; U. Guiñazú, Trad.; Título original: Histoire de la sexualité). México DF, México: Siglo XXI.

Galaz Valderrama, C., Sepúlveda Galeas, M., Poblete Melis, R., Troncoso Pérez, L. \& Morrison Jara, R. (2018). Derechos LGBTI en Chile: tensiones en la constitución de otredades sexualizadas. Psicoperspectivas, 17(1), 1-11. https://doi.org/10.5027/psicoperspectivasvol17-issue1-fulltext-1165

Grzanka, P. R. \& Miles, J. R. (2016). The problem with the phrase "intersecting identities": LGBT affirmative therapy, intersectionality, and neoliberalism. Sexuality Research and Social Policy, 13, 371-389. https://doi.org/10.1007/s13178-016-0240-2

Han, C. (2012). Life in debt: Times of care and violence in neoliberal Chile. Berkeley, CA: University of California Press.

Kuhar, R. \& Paternotte, D. (Eds.) (2017). Anti-gender campaigns in Europe: Mobilizing against equality. Lanham, MD: Rowman \& Littlefield International.

Latham, J. R. (2017). Making and treating trans problems: The ontological politics of clinical practices. Studies in Gender and Sexuality, 18, 40-61. https://doi.org/10.1080/15240657.2016.1238682

Lorenzo, E. (2014). Mi trabajo con AMS. En LoSé [Blog]. Extraído de http://elenalorenzo.com/?p=475

Lugones, M. (2008). Colonialidad y género. Tabula Rasa, 9, 73-101. https://doi.org/10.25058/20112742.340

Mikulak, M. (2018). Telling a poor man he can be rich: Reparative therapy in contemporary Poland. Sexualities. Anticipo en línea de la publicación. https://doi.org/10.1177/1363460718797543 Extraído de https://journals.sagepub.com/doi/abs/10.1177/1363460718797543

Moberly, E. R. (1983). Homosexuality: A new Christian ethic. Cambridge, Reino Unido: James Clarke.

Mol, A. (2006/2008). The logic of care: Health and the problem of patient choice (Peek Language Services \& A. Mol, Trads.; Título original: De logica van het zorgen. Actieve patiënten en de grenzen van het kiezen). New York, NY: Routledge.

Morán Faúndez, J. M. (2012). El activismo católico conservador y los discursos científicos sobre sexualidad: cartografía de una ciencia heterosexual. Sociedad y Religión, 22(37), 167-205. Extraído de http://www.scielo.org.ar/pdf/syr/v22n37/v22n37a06.pdf

Morán, J. M. \& Vaggione, J. M. (2012). Ciencia y religión (hétero)sexuadas: el discurso científico del activismo católico conservador sobre la sexualidad en Argentina y Chile. Contemporânea: Revista de Sociologia da UFSCar, 2(1), 159-185. Extraído de http://www.contemporanea.ufscar.br/index.php/contemporanea/article/download/64/37

Nicolosi, J. (1997). Reparative therapy of male homosexuality: A new clinical approach (2a ed.). Lanham, MD: Rowman \& Littlefield.

Nicolosi, J. (1993/2009). Quiero dejar de ser homosexual: casos reales de terapia reparativa (E. Sánchez Chamizo, Trad.; Título original: Healing homosexuality. Case stories of reparative therapy). Madrid, España: Encuentro.

Nicolosi, J. (s.f.). ¿Qué es la terapia reparativa? Examinando la controversia. Extraído de https://static1.squarespace.com/static/5527394ae4b0ab26ec1c196b/553007bde4b0d72dc645b7ca/553007bde4b0d72dc645b7cc/1397 062233034/la_terapia_reparativa.pdf

Ojeda, T. (2019, Abril 13). Fighting for and against (homo) sexualities in Chile: The case of reparative practices. Engenderings [Blog], Anti-gender. Extraído de https://blogs.lse.ac.uk/gender/2019/04/15/fighting-for-and-against-homo/

Ojeda Güemes, T. (2015). El lugar de la cura en la terapia reparativa con personas homosexuales: análisis crítico de las condiciones de emergencia del dispositivo reparativo y sus implicancias sobre el trabajo clínico con personas homosexuales (Tesis de Magíster, Universidad de Chile, Santiago, Chile). Extraído de http://repositorio.uchile.cl/handle/2250/140788 
Ojeda Güemes, T. \& Avaria, T. (2017, Febrero 20). Ofertas de "cura" para personas LGBT: a propósito de "Arenito". El Desconcierto.cl [Blog], Opinión. Extraído de http://www.eldesconcierto.cl/2017/02/20/ofertas-de-cura-para-personas-lgbt-a-proposito-de-arenito/

Organización Panamericana de la Salud (2012). "Curas" para una enfermedad que no existe: las supuestas terapias de cambio de orientación sexual carecen de justificación médica y son éticamente inaceptables.Washington, DC: Autor. Extraído de http://www.paho.org/hq/index.php?option=com_docman\&task=doc_download\&gid=17704\&Itemid\%20

Ortiz, M. (2017, Diciembre 9). Terapias de reconversión homosexual: iser o no ser? La Tercera, Tendencias. Extraído de http://www2.latercera.com/noticia/terapias-reconversion-homosexual-no/

Pecheny, M. \& de la Dehesa, R. (2014). Sexuality and politics in Latin America: An outline for discussion. En S. Corrêa, R. de la Dehesa \& R. G. Parker (Eds.), Sexuality and politics: Regional dialogues from the global south (Vol. 1, pp. 96-135). Rio de Janeiro, Brasil: Sexuality Policy Watch.

Prieto Damm, C. (2017, Febrero 17). De ocultarse a ser feliz: cómo Santiago pasó de gustarles los hombres a las mujeres. El Demócrata [Blog], Noticias. Extraído de https://www.eldemocrata.cl/noticias/de-ocultarse-a-ser-feliz-como-santiago-paso-de-gustarles-loshombres-a-las-mujeres/

Radiszcz, E. (2009). Sobre el uso de la psicoterapia como dispositivo gubernamental. En H. Cavieres (Ed.), Psicología; ética e ideología (pp. 111-126). Santiago, Chile: Ediciones Universidad Católica Silva Henríquez.

Rich, A. (1981/2001). Heterosexualidad obligatoria y existencia lesbiana (M. S. Sánchez Gómez, Trad.; Título original: Compulsory heterosexuality and lesbian existence). En A. Rich, Sangre, pan y poesía. Prosa escogida: 1979-1985 (M. S. Sánchez Gómez, Trad.; Título original: Blood, bread and poetry) (pp. 41-86). Barcelona, España: Icaria.

Ríos, M. M. (2014, Octubre 3). Marcela Ferrer, la psicóloga que abre esperanza a quienes sufren por sentir atracción al mismo sexo. Portaluz, Temas: Fe y Cultura. Extraído de https://www.portaluz.org/marcela-ferrer-la-psicologa-que-abre-esperanza-a-quienessufren-por-817.htm

Russo, J. A. (2004/2013). From deviance to disorder: The medicalization of sexuality in contemporary psychiatric classifications of disease (J. Longaker, Trad.; Título original: Do desvio ao transtorno: a medicalização da sexualidade na nosografia psiquiátrica contemporânea). En H. Sívori, S. Carrara, J. Russo, M. L. Heilborn, A. P. Uziel \& B. Zilli (Eds.), Sexuality, culture and politics: A South American reader (pp. 655-670). Rio de Janeiro, Brasil: Latin American Center on Sexuality and Human Rights/Institute of Social Medicine/Center for Study and Research in Collective Health.

Sabsay, L. (2012). The emergence of the other sexual citizen: Orientalism and the modernisation of sexuality. Citizenship Studies, 16, 605-623. https://doi.org/10.1080/13621025.2012.698484

Schroeder, M. \& Shidlo, A. (2002). Ethical issues in sexual orientation conversion therapies: An empirical study of consumers. Journal of Gay \& Lesbian Psychotherapy, 5(3-4), 131-166. https://doi.org/10.1300/J236v05n03_09

Sedgwick, E. K. (1992/1993). Epidemics of the will. En E. K. Sedgwick, Tendencies (pp. 129-140). Durham, NC: Duke University Press.

Solari, C. (2010, Octubre 22). Reconversión gay Paula [Revista], Reportajes y Entrevistas. Extraído de http://www.paula.cl/reportajesy-entrevistas/reconversion-gay/

Stein, E. (1999). The mismeasure of desire: The science, theory, and ethics of sexual orientation. New York, NY: Oxford University Press.

Suess, A. (2014). Cuestionamiento de dinámicas de patologización y exclusión discursiva desde perspectivas trans e intersex. Revista de Estudios Sociales, 49, 128-143. https://doi.org/10.7440/res49.2014.10

van den Aardweg, G. J. M. (1985/2005). Homosexualidad y esperanza: terapia y curación en la experiencia de un psicólogo (J. Torrents, Trad.; Título original: Homosexuality and hope: A psychologist talks about treatment and change). Pamplona, España: Ediciones Universidad de Navarra.

Viveros Vigoya, M. \& Rodríguez Rondón, M. A. (2017). Hacer y deshacer la ideología de género. Sexualidad, Salud y Sociedad, 27, 118127. https://doi.org/10.1590/1984-6487.sess.2017.27.07.a

Waidzunas, T. (2015). The straight line: How the fringe science of ex-gay therapy reoriented sexuality. Minneapolis, MN: University of Minnesota Press.

Wilkinson, A. K. (2013). "Sin sanidad, no hay santidad". Las prácticas reparativas en Ecuador. Quito, Ecuador: Facultad Latinoamericana de Ciencias Sociales.

World Health Organization (2018). ICD-11: Classifying disease to map the way we live and die. Washington, DC: Autor. Extraído de http://www.who.int/health-topics/international-classification-of-diseases

Zegers, B., Larraín, M. E. \& Bustamante, F. (Eds.) (2007). Sobre la homosexualidad. Santiago, Chile: Mediterráneo.

Fecha de recepción: Agosto de 2018.

Fecha de aceptación: Agosto de 2019. 